\title{
Análise da participação das mulheres no projeto da IX Semana Acadêmica da Engenharia Mecânica (SAEMEC)
}

\author{
Francisco Daniel França Bazán ${ }^{1}$, Tatiana Annoni Pazeto² \\ ${ }^{1}$ Curso de Bacharelado em Engenharia Mecânica - Universidade Federal de Rondonópolis \\ (UFR) \\ ${ }^{2}$ Curso de Bacharelado em Sistemas de Informação - Universidade Federal de Rondonópolis \\ (UFR) \\ 78735-901 - Rondonópolis - MT- Brasil \\ francisco.danieldaluno.ufr.edu.br, tpazeto@gmail.com
}

\begin{abstract}
Gender equality is a relevant issue and in some fields, such as Science, Technology, Engineering and Mathematics (STEM), it does not always occur. Therefore, it is important to analyze the female presence at an event in this field. In this context, this article aims to analyze the female participation in the IX SAEMEC extension project, comparing it with the number of women enrolled in the Mechanical Engineering course at UFR. For this, the reports of the students enrolled in that course and that of the IX SAEMEC were analyzed, as well as the national theoretical framework on the subject. It was concluded that the number of men is greater in the exact sciences field, which also occurs in the Mechanical Engineering course at UFR and in the participation of women in the IX SAEMEC event.
\end{abstract}

Resumo. A igualdade de gênero é um tema relevante e em algumas áreas, como Ciência, Tecnologia, Engenharia e Matemática (STEM), nem sempre ocorre. Portanto é importante analisar a presença feminina em um evento dessa área. Nesse contexto, este artigo visa analisar a participação feminina no projeto de extensão da IX SAEMEC comparando com a quantidade de mulheres matriculadas no curso de Engenharia Mecânica da UFR. Para isso, analisou-se o relatório dos alunos matriculados no referido curso e o da IX SAEMEC, bem como o referencial teórico nacional sobre o tema. Concluiu-se que a quantidade de homens ainda é maior na área de exatas, o que também ocorre no curso de Engenharia Mecânica da UFR e na participação de mulheres no evento da IX SAEMEC.

\section{Introdução}

Há investigações e pesquisas sobre a participação feminina em diversos setores, sendo que a igualdade de gênero é fundamental. No entanto, ainda há preconceito quanto a atuação da mulher em alguns cargos, funções e profissões.

Neste sentido, o presente artigo visa analisar a participação feminina em um projeto de extensão da engenharia mecânica, bem como o quantitativo de mulheres que estão vinculadas ao referido curso.

Para isso, vários documentos do curso foram analisados, tais como o número de alunos matriculados, bem como a participação das mulheres no projeto de extensão da IX Semana Acadêmica da Engenharia Mecânica (SAEMEC). Também foi feito um levantamento à nível nacional da atuação feminina em alguns seguimentos. 
O trabalho está estruturado em cinco seções. A seção 2 versa sobre a atuação feminina em alguns setores da economia. A seguinte, aborda a atuação feminina em cursos da área de exatas. A análise da participação e atuação feminina no referido projeto é discorrida na seção 4. Por fim, as conclusões são abordadas.

\section{Indicadores da Atuação Feminina}

Mesmo a América Latina sendo considerada a região que mais avançou nas questões de igualdade de gênero no mundo, de acordo com o Ranking do Fórum Econômico Mundial de 2018, que analisa a desigualdade de gênero em 149 países, o Brasil ainda se encontra na 90a posição [Costa; Tombesi, 2019].

Segundo o Instituto Brasileiro de Geografia e Estatística (IBGE) (IBGE, 2020), em 2019, as mulheres compunham cerca de $51,8 \%$ da população do país. Porém, observando-se a inclusão dessa fatia nos indicadores de saúde, trabalho, vida pública e educação ainda é notável a desigualdade entre gêneros presente no território brasileiro. Já o IBGE (2018) mostra que, no âmbito da vida pública, os cargos gerenciais e assentos na câmara dos deputados exercidos por mulheres são de apenas 39,1\% e 10,5\%, respectivamente.

Já na educação a situação muda completamente. Observando as taxas de frequência escolar e conclusão de ensino superior, as mulheres encontram-se percentualmente acima dos homens, com $73,5 \%$ contra $63,2 \%$ e $23,5 \%$ contra $20,7 \%$, respectivamente [Fundação Tide Setubal, 2018]. Ou seja, mulheres tendem a gerar mais resultados na educação. Apesar de serem maioria na educação, segundo dados da Unesco Brasil (2018), o público feminino ainda não possui participação numérica expressiva no âmbito de STEM, compondo apenas $33,1 \%$ dos graduados na área [IBGE, 2018]. O fato também é corroborado, de algum modo, por Prietch, Pazeto e Souza (2012), que versa sobre um curso na área de Informática.

\section{Mulheres em Ciência, Tecnologia, Engenharia e Matemática (STEM)}

Conforme a Unesco Brasil (2018) sobre o incentivo de mulheres em STEM, existem quatro âmbitos que influenciam na sua participação, avanço e desempenho nessas áreas, sendo eles: individuais, familiares, escolares e sociais. Esses mesmos fatores também são referentes ao Mau Desempenho Escolar (MDE), relatado por Siqueira e Gurgel-Giannetti (2011).

No contexto escolar, segundo Unesco Brasil (2018), docentes qualificados tendem a influenciar de forma positiva o envolvimento de meninas na área de STEM. Se forem docentes mulheres, os benefícios são ainda maiores para as meninas, provavelmente, por atuarem como exemplos e anularem estereótipos de gênero relativos à área. Além disso, práticas, experiências, estágios e aconselhamentos profissionais são capazes de interessar e expandir a percepção das garotas nas áreas de STEM. Aquino (1998) relata que, apesar das mudanças ao longo dos anos, ainda há muito preconceito com relação às escolhas profissionais e a influência do professor nesse quesito.

No ambiente social, a cultura e a sociedade em que cada mulher está inserida contribui para a percepção de suas habilidades, papéis, carreiras e aspirações de vida. O grau de igualdade de gênero de uma sociedade influencia no número de mulheres presentes nas áreas de STEM. Além disso, legislações, políticas públicas de integração de gênero e a mídia podem perpetuar ou neutralizar os estereótipos de gênero sobre habilidades e carreiras nas áreas de STEM [Moraes,2016]; [Unesco Brasil,2018]; [Uamusse; Cossa; Kouleshova, 2020].

A Tabela 1, adaptada de Inep/MEC (2019), apresenta os 20 maiores cursos de graduação com alunos matriculados. 
Tabela 1. Os 20 maiores cursos de graduação em número de matrículas e os respectivos percentuais de participação, por sexo - Brasil - 2017

\begin{tabular}{|c|c|c|c|c|c|c|}
\hline \multicolumn{7}{|c|}{ Os 20 maiores cursos de graduação em número de matrículas e os respectivos percentuais de participação, por sexo } \\
\hline \multicolumn{2}{|r|}{ Cursos de Graduação } & $\begin{array}{r}\text { Total de } \\
\text { Inscritos }\end{array}$ & $\begin{array}{l}\text { Mulheres } \\
\text { Inscritas }\end{array}$ & $\begin{array}{l}\text { Percentual de } \\
\text { Mulheres }\end{array}$ & $\begin{array}{l}\text { Homens } \\
\text { Inscritos }\end{array}$ & $\begin{array}{l}\text { Percentual } \\
\text { de Homens }\end{array}$ \\
\hline \multicolumn{2}{|r|}{ Ciências Exatas } & 809.394 & 288.294 & $35,6 \%$ & 521.100 & $64,4 \%$ \\
\hline & Engenharia Civil & 346.827 & 105.782 & $30,5 \%$ & 241.045 & $69,5 \%$ \\
\hline $9^{\circ} A_{-1}$ & Arquitetura e Urbanismo & 168.291 & 112.082 & $66,6 \%$ & 56.209 & $33,4 \%$ \\
\hline $11^{\circ} \mathrm{E}$ & Egenharia de Produção & 162.427 & 56.849 & $35,0 \%$ & 105.578 & $65,0 \%$ \\
\hline \begin{tabular}{l|l}
$16^{\circ}$ & $\mathrm{E}$ \\
\end{tabular} & Engenharia Mecânica & 131.849 & 13.580 & $10,3 \%$ & 118.269 & $89,7 \%$ \\
\hline & Ciências Humanas e Biológicas & 1.464 .378 & 996.490 & $68,0 \%$ & 467.888 & $32,0 \%$ \\
\hline \begin{tabular}{l|l}
$6^{\circ}$ & $\mathrm{E}$ \\
\end{tabular} & Enfermagem & 295.126 & 247.906 & $84,0 \%$ & 47.220 & $16,0 \%$ \\
\hline \begin{tabular}{l|l}
$7^{\circ}$ & $P$ \\
\end{tabular} & Psicologia & 149.956 & 120.715 & $80,5 \%$ & 29.241 & $19,5 \%$ \\
\hline \begin{tabular}{l|l}
$8^{\circ}$ & $P$ \\
\end{tabular} & Prof. de Educação Física & 185.792 & 74.688 & $40,2 \%$ & 111.104 & $59,8 \%$ \\
\hline $10^{\circ} \mathrm{F}$ & Fisioterapia & 164.016 & 129.573 & $79,0 \%$ & 34.443 & $21,0 \%$ \\
\hline $14^{\circ} \mathrm{N}$ & Medicina & 149.655 & 87.099 & $58,2 \%$ & 62.556 & $41,8 \%$ \\
\hline $15^{\circ} \mathrm{E}$ & Educação Física & 148.151 & 51.853 & $35,0 \%$ & 96.298 & $65,0 \%$ \\
\hline $17^{\circ} \mathrm{N}$ & Nutrição & 128.343 & 109.348 & $85,2 \%$ & 18.995 & $14,8 \%$ \\
\hline $18^{\circ} \mathrm{F}$ & Farmácia & 127.633 & 91.768 & $71,9 \%$ & 35.865 & $28,1 \%$ \\
\hline $19^{\circ} \mathrm{C}$ & Odontologia & 115.706 & 83.540 & $72,2 \%$ & 32.166 & $27,8 \%$ \\
\hline & Ciencias Humanas & 3.058 .529 & 2.041 .139 & $66,7 \%$ & 1.017 .390 & $33,3 \%$ \\
\hline \begin{tabular}{l|l}
$1^{\circ}$ & $\mathrm{L}$ \\
\end{tabular} & Direito & 879.234 & 486.216 & $55,3 \%$ & 393.018 & $44,7 \%$ \\
\hline $2^{\circ} \mathrm{P}$ & Pedagogia & 714.345 & 660.769 & $92,5 \%$ & 53.576 & $7,5 \%$ \\
\hline \begin{tabular}{l|l}
$3^{\circ}$ & $A$ \\
\end{tabular} & Administração & 682.555 & 374.723 & $54,9 \%$ & 307.832 & $45,1 \%$ \\
\hline $4^{\circ} \mathrm{C}$ & Ciências Contábeis & 362.042 & 206.364 & $57,0 \%$ & 155.678 & $43,0 \%$ \\
\hline $12^{\circ} \mathrm{C}$ & Gestão de Pessoas & 156.978 & 122.443 & $78,0 \%$ & 34.535 & $22,0 \%$ \\
\hline $13^{\circ} \mathrm{S}$ & Serviço Social & 153.548 & 138.347 & $90,1 \%$ & 15.201 & $9,9 \%$ \\
\hline $20^{\circ} \mathrm{E}$ & Empreendedorismo & 109.827 & 52.278 & $47,6 \%$ & 57.549 & $52,4 \%$ \\
\hline & Total & 5.332 .301 & 3.325 .923 & $62,4 \%$ & 2.006 .378 & $37,6 \%$ \\
\hline
\end{tabular}

Fonte: Própria adaptada com base em Inep/MEC (2019)

Analisando a Tabela 1 percebe-se que os quatro primeiros cursos com maior número de alunos matriculados são da área de ciências humanas, sendo que os mesmos também têm uma quantidade maior de mulheres comparando com o quantitativo de homens. No entanto, o quinto curso em número de matrículas é Engenharia Civil, mas a quantidade de homens é quase o dobro. Outra constatação é que a maioria dos cursos de ciências biológicas tem um público feminino superior ao masculino. Outrossim, dos vinte mais procurados, apenas quatro são de ciências exatas, sendo um deles de Engenharia Mecânica. Contudo, o quantitativo de mulheres é extremamente baixo.

O trabalho de Hill, Corbett e St. Rose (2010) corrobora os dados apresentados, relatando que na graduação, os homens superam as mulheres em quase todos os campos de STEM. Em graduações como física, engenharia e ciência da computação, a diferença é expressiva, na qual apenas $20 \%$ das mulheres concluem o curso. O mesmo ocorre em um curso de Licenciatura em Informática abordado em Prietch, Pazeto e Souza (2012).

Os dados da tabela anterior também refletem o exposto por Oliveira, Unbehaum e Gava (2019, p.3) no qual é mencionado que as mulheres concentram-se em "áreas de educação (pedagogia) e da saúde (enfermagem e atenção primária)".

Conforme o Inep/MEC (2019), entre os 20 maiores cursos de graduação em número de matrículas no país, os cursos de Engenharia Civil, Engenharia de Produção e Engenharia Mecânica possuem menos mulheres em comparação aos demais cursos.

$\mathrm{Na}$ Tabela 1 nota-se que a participação feminina (62\%) é superior à masculina (38\%) consoante ao número de matrículas em curso de graduação, o que corrobora com Unesco Brasil (2018). Porém, ao separar as áreas de conhecimento em exatas, humanas e biológicas, nota-se que a proporção se inverte, com $64,4 \%$ de homens e apenas $35,6 \%$ de mulheres na área de exatas. A engenharia mecânica é o curso com o menor percentual de participação feminina entre os vinte, com apenas $10,3 \%$ de matrículas. Vale salientar que os percentuais se referem ao total dos cursos. 


\section{Quantitativo de Mulheres nos Cursos de Engenharia Mecânica da IX Semana Acadêmica da Engenharia Mecânica (SAEMEC)}

A SAEMEC é um evento anual do curso de Engenharia Mecânica da UFR organizado por alunos e professores. Geralmente ocorre em cinco dias e abrange diversas atividades, como palestras, minicursos, visitas técnicas, competições, apresentação de projetos desenvolvidos ou em desenvolvimento para alunos do ensino fundamental, entre outras.

A Tabela 2 apresenta a relação de alunos matriculados no curso, separados por sexo. Os dados foram disponibilizados pelo Registro Escolar, sendo que no arquivo não constava o campo sexo. Através dos nomes, os mesmos foram qualificados, pelos autores, quanto ao sexo. Já os dados da IX SAEMEC foram obtidos através do relatório final do projeto, o qual foi aprovado pelas instâncias superiores. Devido à falta do campo sexo, em ambos os formulários, para a análise e tabulação de dados, erros podem ter sido cometidos. Salienta-se que quando haviam dúvidas do nome, alguns colegas e professores foram questionados.

\section{Tabela 2. Número e proporção de alunos matriculados no curso de Engenharia Mecânica da UFR no segundo semestre de 2019

\begin{tabular}{|r|c|c|}
\hline \multicolumn{2}{|c|}{ Total de Alunos Matriculados } \\
\hline Sexo/ Total & Quantidade & Percentual \\
\hline Homens & 386 & $86,55 \%$ \\
\hline Mulheres & 60 & $13,45 \%$ \\
\hline Total de Alunos Matriculados & 446 & $100,00 \%$ \\
\hline
\end{tabular}

Conforme a Tabela 2, no semestre de 2019/2, havia 446 alunos matriculados no curso. Desses, 386 (86,55\%) homens e apenas 60 (13,45\%) mulheres. Analisando os dados da Tabela 1 em que a participação feminina era de 10,3\% no curso de Engenharia Mecânica, no mesmo curso a UFR apresenta participação feminina ligeiramente superior. Analisando apenas o curso de Engenharia Mecânica da UFR comparado ao percentual de mulheres matriculadas na Tabela $1(10,3 \%)$, o número de alunas matriculadas é superior em $3,15 \%$. No entanto, nota-se que a distância entre o número de homens e mulheres, estudantes do citado curso, ainda é muito desigual, com a proporção de uma mulher para cada seis homens (1/6).

A Tabela 3 apresenta o total de alunos inscritos na IX SAEMEC com as respectivas proporções separadas por sexo.

Tabela 3. Proporção de Homens e Mulheres inscritos no evento

\begin{tabular}{|r|c|c|}
\hline \multicolumn{3}{|c|}{ Participantes da IX SAEMEC } \\
\hline Sexo & Quantidade & Percentual \\
\hline Homens & 73 & $86,90 \%$ \\
\hline Mulheres & 11 & $13,10 \%$ \\
\hline Total & 84 & $100,00 \%$ \\
\hline
\end{tabular}

A respeito da IX SAEMEC, constatou-se que, dos 84 participantes aptos a receberem certificados do evento, apenas $11(13,1 \%)$ eram mulheres, proporção muito próxima à de alunas matriculadas no curso. Portanto, pode-se mencionar que a taxa de mulheres e homens, dentro do grupo de inscritos no evento, repetiu a proporção observada no número total de mulheres e homens regularmente matriculados no curso (Tabela 2). No entanto, considerando o número de alunos matriculados, a participação no evento é muito pequena.

A Tabela 4 apresenta a proporção entre o número de inscritos na IX SAEMEC confrontando com o número de matriculados no curso.

Tabela 4. Proporção dos inscritos na IX SAEMEC em relação aos totais de homens e mulheres matriculados no curso

\begin{tabular}{|c|c|c|}
\hline \multicolumn{3}{|c|}{ Proporção dos inscritos na IX SAEMEC em relação aos matriculados no curso } \\
\hline Sexo / Total & Quantidade & Percentual \\
\hline Homens & 73 & $18,91 \%$ \\
\hline Mulheres & 11 & $18,33 \%$ \\
\hline Total & 84 & $18,83 \%$ \\
\hline
\end{tabular}


Comparando os dados da Tabela 4 com a Tabela 2, consoante às mulheres, pode-se perceber que o número de inscritas na IX SAEMEC (11) e matriculadas no curso, 18,3\% delas participaram do evento. De modo similar, dos 386 homens matriculados, 18,9\% participaram do evento, compondo um total de 73 participantes. Isso significa que quanto à participação no evento, há equivalência entre os gêneros.

A participação das mulheres em minicursos e visitas técnicas consta na Tabela 5.

\begin{tabular}{|c|c|c|c|c|}
\hline MINICURSOS & Homens & Mulheres & Total & Participação fem \\
\hline Controlador Lógico Programável (CLP) & 3 & 0 & 3 & $0 \%$ \\
\hline Veículo Aéreo Remotamente Pilotado (VARP) - "Drones" & 5 & 0 & 5 & $0 \%$ \\
\hline Fundição & 4 & 1 & 5 & $20 \%$ \\
\hline LaTeX & 2 & 0 & 2 & $0 \%$ \\
\hline Manutenção Hidráulica e Pneumática & 8 & 1 & 9 & $11 \%$ \\
\hline Matlab & 4 & 2 & 6 & $33 \%$ \\
\hline Oratória & 8 & 1 & 9 & $11 \%$ \\
\hline Phyton & 3 & 1 & 4 & $25 \%$ \\
\hline Robôs Lego & 12 & 2 & 14 & $14 \%$ \\
\hline TOTAL & 49 & 8 & 57 & $14 \%$ \\
\hline VISITAS TÉCNICAS & Homens & Mulheres & Total & Participação fem \\
\hline Cargill & 9 & 5 & 14 & $36 \%$ \\
\hline Cooperfibra & 10 & 3 & 13 & $23 \%$ \\
\hline Engie Brasil & 16 & 4 & 20 & $20 \%$ \\
\hline Grupo Petrópolis & 19 & 3 & 22 & $14 \%$ \\
\hline Inst. MT algodão & 16 & 4 & 20 & $20 \%$ \\
\hline Raízen & 6 & 2 & 8 & $25 \%$ \\
\hline Rumo & 20 & 3 & 23 & $13 \%$ \\
\hline Usina Sonora & 13 & 1 & 14 & $7 \%$ \\
\hline TOTAL & 109 & 25 & 134 & $19 \%$ \\
\hline
\end{tabular}

Devido à necessidade de ônibus para o transporte até os locais das visitas técnicas e pré-requisitos e/ou limitações dos recursos para a execução dos minicursos, os primeiros inscritos tinham prioridade de escolha. Outrossim, a participação nessas modalidades poderia ser de, no máximo, oito horas ( $8 \mathrm{~h}$ ). Isso significa que, se um minicurso, por exemplo, fosse de oito horas (8h), o inscrito só poderia fazer esse. No entanto, não há informações no relatório sobre a ordem de inscrições para saber se isso influenciou na participação feminina.

Analisando os dados da Tabela 5, pode-se constatar que tanto as mulheres quanto os homens preferem as visitas técnicas e que em todas as atividades a participação feminina é inferior a masculina. Isso ocorre porque o número de mulheres matriculadas é inferior ao de homens, conforme as Tabela 2 e 3.

Quanto aos minicursos, não houve inscrições de mulheres nos minicursos de Controlador Lógico Programável (CLP), "Drones" e LaTeX e que a preferência feminina é por minicursos na área de programação (Matlab e Phyton), sendo essa de $29 \%$. Considerando a proporção de inscritos, apresentada na Tabela 4, a maioria dos minicursos não atingiu o percentual de 18,33\%. Isso só ocorreu nos minicursos de Fundição, Matlab e Phyton.

Consoante às visitas técnicas, em todas houve participação feminina, sendo maior nas visitas à Cargill, Instituto Mato-Grossense do Algodão (IMA-MT) e ENGIE Brasil se comparada as demais empresas. Analisando o percentual da participação das mulheres nas visitas, apenas às visitas realizadas a Usina Sonoro, Rumo e Grupo Petrópolis não atingiram o percentual de inscritas, mencionado na Tabela 4. Isso pode ser um reflexo da limitação de participantes em visitas técnicas e minicursos, bem como da carga horária dessas atividades.

O número de alunos que organizaram a IX SAEMEC é apresentado na Tabela 6.

Tabela 6. Número de discentes organizadores da IX SAEMEC

\begin{tabular}{|c|c|c|}
\hline \multicolumn{3}{|c|}{ Composição da Equipe SAEMEC } \\
\hline Sexo / Total & Quantidade & Percentual \\
\hline Homens & 26 & $84 \%$ \\
\hline Mulheres & 5 & $16 \%$ \\
\hline Total & $\mathbf{3 1}$ & $\mathbf{1 0 0} \%$ \\
\hline
\end{tabular}


Quanto à equipe organizadora do evento, dentre os 31 integrantes discentes, apenas cinco eram mulheres (16\%), proporção que não se distingue de forma significativa dos demais dados com relação ao número de estudantes mulheres no curso e no número de participantes mulheres no evento. No entanto, é importante ressaltar que a coordenadora do projeto, bem como a presidente dos alunos era do sexo feminino.

A partir dos dados apresentados, observou-se que os números referentes à presença feminina, tanto no curso de Engenharia Mecânica quanto no evento da IX SAEMEC, indicam um aumento no número de mulheres em relação ao cenário nacional do último Censo [Inep/MEC, 2019] e que sua participação no evento acadêmico é proporcional ao número de estudantes mulheres do curso.

\section{Conclusões}

No presente artigo foi analisada a participação feminina na IX SAEMEC considerando o quantitativo de mulheres que estão vinculadas ao referido curso, bem como um comparativo quanto à participação feminina em cursos da área de exatas.

Através dos dados analisados na seção 4 comprovou-se que a atuação feminina ainda é pequena, se comparada aos homens, no curso de Engenharia Mecânica da UFR. Isso ocorre em todas as áreas relacionadas ao STEM, descritos na seção 3. No entanto, em relação à IX SAEMEC, a participação feminina é de $18,33 \%$ (Tabela 4), sendo superior ao número de alunas matriculadas no curso de Engenharia Mecânica (10,3\%) exposto na Tabela 1.

Também pode-se constatar que, conforme o texto da seção 3 , ainda há baixa procura feminina por cursos da área de exatas.

Para a realização do trabalho, como a maior parte dos dados não estão cadastrados em um sistema, toda a tabulação dos mesmos foi realizada manualmente, o que gerou morosidade. Contudo, obteve-se êxito e o trabalho atingiu os objetivos almejados. Além disso, a revisão da literatura corrobora com a análise realizada.

Em trabalhos futuros pode ser feito um comparativo com as demais edições da SAEMEC, considerar o quantitativo de formados, desistentes e em trancamento, realizar análise em outros eventos de Engenharia Mecânica, bem como verificar se a participação feminina na área de exatas está aumentando. Também pode-se investigar se os fatores mencionados pela Unesco Brasil (2018) e Siqueira e Gurgel-Giannetti (2011) influenciam na baixa procura das mulheres em cursos de exatas.

\section{Referências}

Aquino, J. C. Diferenças e preconceitos na escola. Alternativas teóricas e práticas. $4^{\circ}$ ed. São Paulo: Summus Editorial, 1998.

Brenner-Shuman, A.; Waren, W. "Age at menarche and choice of college major: implications for STEM Majors.” Bulletin of Science, Technology \& Society, v. 33, n. 1-2, p. 28-34, 2013.

Calegari, L. (2018) "A desigualdade de gênero no Brasil em um gráfico". Disponível em: https://exame.abril.com.br/brasil/a-desigualdade-de-genero-no-brasil-em-um-grafico/. Acesso em: janeiro/2021

Costa, C.; Tombesi, C. "Dia Internacional da Mulher: 6 gráficos que mostram como as mulheres avançaram (ou não) na América Latina." Da BBC News Brasil e da BBC News 
Mundo em Londres. Março/2019. Disponível em: https://www.bbc.com/portuguese/brasil47490977. Acesso em: janeiro/2021

Freire, P. Conscientização: teoria e prática da libertação. 3. ed. São Paulo: Centauro, 1980.

Fundação Tide Setubal. (2018). "Desigualdade de gênero no Brasil: uma realidade perigosa.". Disponível em: <https://fundacaotidesetubal.org.br/desigualdade-de-genero-no-brasiluma-realidade-perigosa/\#: : :text=Pensando $\% 20 \mathrm{em} \% 20$ termos $\% 20$

populacionais $\% 2 \mathrm{C} \% 20 \mathrm{as}, \% 2 \mathrm{C} 48 \% 25 \% 20 \mathrm{da} \% 20$ população $\% 20$ nacional.\&text $=\mathrm{O} \% 20 \mathrm{fem}$ inicídio\%20é\%20tão\%20frequente,Mundial\%20da\%20Saúde $\% 20(\mathrm{OMS})>$. Acesso em: março/2021.

Hill, C.; Corbett, C.; St. Rose, A. Why so few women in Science, Technology, Engineering and Mathematics. Washington DC: American Association of University Women, 2010.

IBGE. (2018). "Estatísticas de Gênero Indicadores sociais das mulheres no Brasil". ISBN 978-85-240-4448-9. Disponível em: https://biblioteca.ibge.gov.br/visualizacao/ livros/liv101551_informativo.pdf. Acesso em: janeiro/2021

IBGE. (2020). "Pesquisa Nacional por Amostra de Domicílios Contínua 2012-2019”. ISBN 978-85-240-4530-1. Disponível em: https:/educa.ibge.gov.br/jovens/conheca-obrasil/populacao/18320-quantidade-de-homens-e-mulheres.html. Acesso em: janeiro/2021

Inep/MEC. (2019). "Resumo Técnico Censo da Educação Superior 2017”. Disponível em: http://portal.inep.gov.br/informacao-da-publicacao/-/asset_publisher/ 6JYIsGMAMkW1/document/id/6725796. Acesso em: fevereiro/2021.

Moraes, A. Z. "Relações de gênero e a formação de engenheiras e engenheiros". Dissertação (Mestrado em Educação) - Universidade do Sul de Santa Catarina, Tubarão, 2016.

Oliveira, E. R. B.; Unbehaum, S.; Gava, T. "A educação STEM e gênero: uma contribuição para o debate brasileiro". Cad. Pesqui. [online]. 2019, vol.49, n.171, pp.130-159. Epub May 20, 2019.

Prietch, S. S.; Pazeto, T. A.; Souza, N. S. “Análise da Atuação das Mulheres em um Curso de Licenciatura em Informática”. In: Anais da XII WEIBASE/ ERBASE (Escola Regional de Computação - Bahia Alagoas Sergipe), Juazeiro/BA: SBC/ Univasf, 2012. p. 1-10.

Sapienza, P.; Zingales, L.; Maestripieri, D. "Gender differences in financial risk aversion and career choices are affected by testosterone". Proceedings of the National Academy of Science, USA, v. 106, n. 36, p. 15268-15273, 2009.

Siqueira, C. M.; Gurgel-Giannetti, J. "Mau desempenho escolar: uma visão atual". Revista da Associação Médica Brasileira Print version. ISSN 0104-4230. Rev. Assoc. Med. Bras. vol.57 no.1 São Paulo Jan./Feb. 2011.

Uamusse, A. A.; Cossa, E. F. R.; Kouleshova, T. A mulher em cursos de ciências, tecnologia, engenharia e matemática no ensino superior moçambicano. Revista Estudos Feministas. Print version. ISSN 0104-026X On-line version. ISSN 1806-9584. Rev. Estud. Fem. vol.28 no.1 Florianópolis, 2020.

Unesco Brasil 2018. (2018). "Decifrar o código: educação de meninas e mulheres em ciências, tecnologia, engenharia e matemática (STEM)". Disponível em: https://ead2.iff.edu.br/pluginfile.php/138994/mod_resource/content/1/Decifrar\%20o\%20C ODIGO.pdf. Acesso em: fevereiro/2021. 